\title{
Modern approaches to multiple sclerosis
}

\author{
Martin Wilson
}

This article will summarise aspects of 'modern approaches to multiple sclerosis (MS)', focusing on areas where there has been significant change in practice within the last decade, and in particular the new therapeutic agents available for relapsing MS which have posed new dilemmas for neurologists prescribing disease modifying therapies (DMT) for the condition.

\section{The basics: aetiology, pathology, and classification}

MS is a common cause of neurological disability in young and middle age adults, second only to trauma in the UK. Approximately 80,000 individuals in the UK have MS. MS is considered to be a predominantly autoimmune, T-cell mediated disease occurring in genetically predisposed individuals after one or more environmental 'triggers'. The identity of the trigger(s) remains unknown, but currently favoured candidates include childhood Epstein-Barr virus infection, and low vitamin D levels. The result is the onset, usually in adulthood, of lesions affecting the white matter of the central nervous system (CNS), as in the brain, spinal cord and optic nerves. The pathology is characterised by demyelination and axonal loss in the CNS white matter (Fig 1). ${ }^{1}$

MS is classified into subtypes according to a simple, but useful scheme: it is purely clinical, based upon the observations of the patient's pattern of disease (Fig 2).

Most patients present with a relapsing-remitting disease (RRMS), with relapses ('attacks') followed by recovery (partial or complete), with marked variability in both severity of relapses, and interval between them. A large proportion of patients with RRMS will eventually develop 'secondary progressive' disease (SPMS), characterised by a reduction or cessation of acute relapses but instead a gradual worsening of disability (Fig 2). The length of time spent in the relapsing phase is markedly variable, but once secondary progression is established the rate of progression is fairly predictable, and essentially irreversible. ${ }^{2}$ There are no DMT for progressive forms of MS.

\section{Diagnosis of multiple sclerosis}

The diagnosis of MS (in its relapsing forms) has long been based upon clinical observation of 'lesions disseminated in both space and time': that is to say, symptoms and signs (consistent with inflammatory demyelination) occurring in different areas of the CNS and at different times (for example an

Martin Wilson, consultant neurologist, The Walton Centre Foundation Trust, Liverpool optic neuritis in 2001 and partial myelitis in 2003). The diagnosis is usually confirmed by typical abnormalities on magnetic resonance imaging (MRI) of the brain (Fig 3) and/or cord: other investigations including lumbar puncture, evoked responses and blood testing can all be useful if the clinical or radiological presentation is atypical.

The current 'McDonald criteria'3 have introduced one significant change to the way we can confirm a diagnosis: although still crucial to demonstrate 'dissemination in time', this can now be done radiologically rather than clinically. In other words, following a first attack of demyelination (for example optic neuritis), and with an abnormal MRI scan, the clinician and patient have two choices: 'wait and see' (if and when a second clinical attack occurs), or repeat the MRI after an interval, eg six months. If the repeat scan shows new lesions, a diagnosis of MS is confirmed even if no further clinical episodes have occurred. The value of this new approach is debatable, as it does not change treatment strategies (at least in the UK) though increasingly patients offered the choice tend to ask for the repeat scan.

\section{Disease modifying therapies in relapsing multiple sclerosis}

The currently available standard DMTs for relapsing MS include Interferon $\beta$ (IFN) and glatiramer acetate (GA). In recent years,

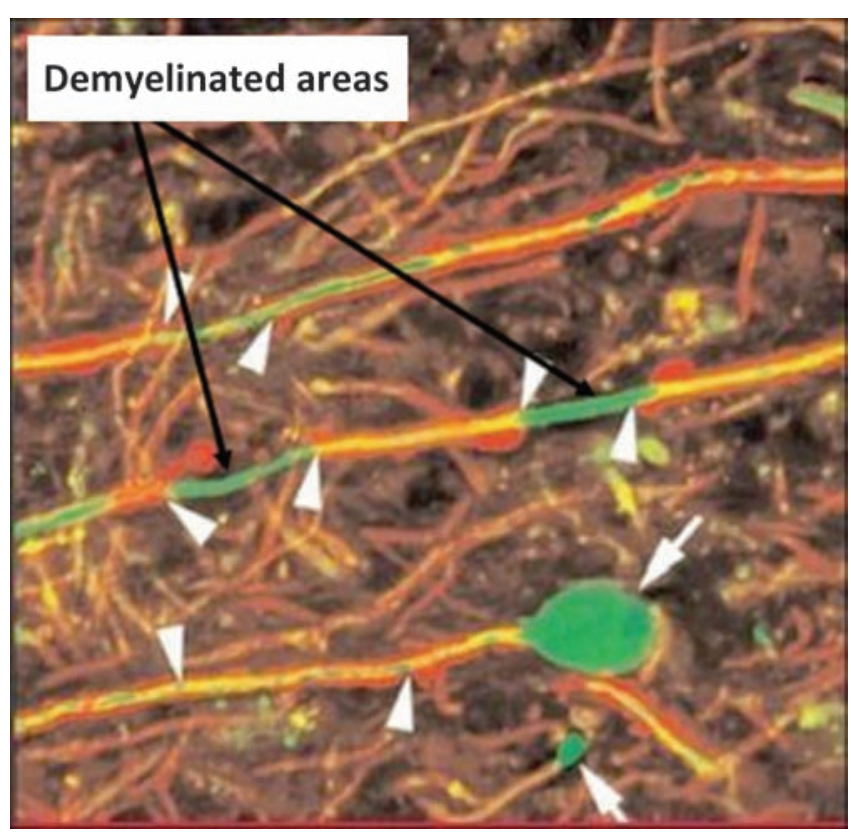

Fig 1. The characteristic lesions of MS. Areas of demyelination (arrow heads) and also axonal transection with formation of 'axonal spheroids' (arrows). 
several more potent agents have become available for patients with either 'aggressive' disease or continuing relapses on standard therapy (Table 1). IFN and GA are self-injected, either subcutaneously or intramuscularly, and usually well tolerated with virtually no serious side effects. They reduce relapses by approximately $30 \%$, though evidence they truly affect the long-term outcome of MS is sparse. ${ }^{4,5}$ These drugs have also been trialled in patients who have experienced a single attack or 'clinically isolated syndrome' (CIS) (ie before MS has been confirmed), in the hope treatment would delay or even prevent the development of MS. All these studies showed similar results: a statistically significant delay in the time to development of MS. Sounds impressive? Well, it is already known that these drugs reduce the rate of relapse (in established MS), and the definition of conversion from CIS to MS is relapse: so this is exactly the result we should have expected. There is no evidence that these drugs can stop a patient destined to develop MS from doing so. The more important question is, do the proportion of CIS patients who go on to develop MS do better in the long run if they were started

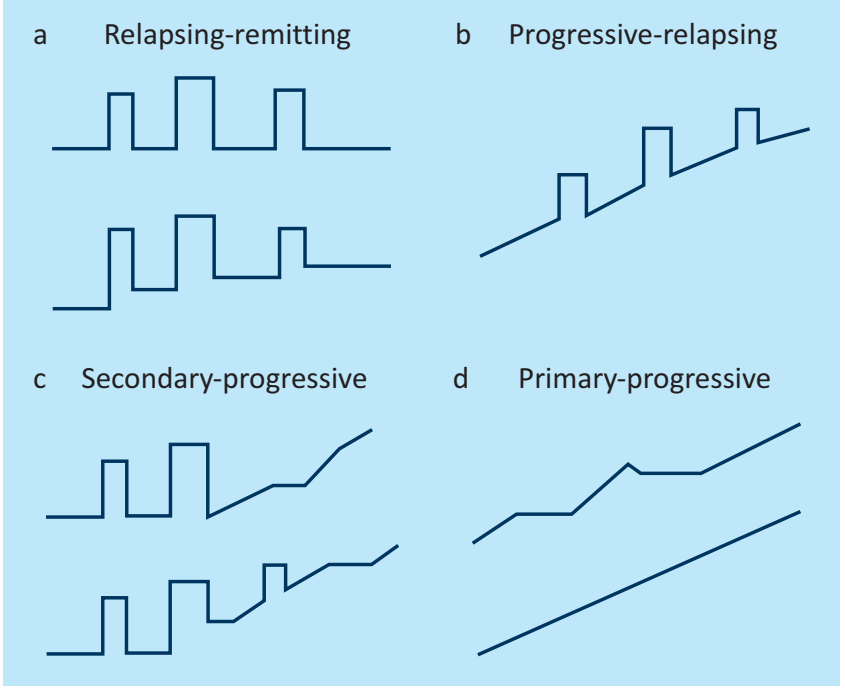

Fig 2. Clinical patterns of multiple sclerosis subtypes: most patients present with a relapsing remitting disorder (a), though many will eventually enter 'secondary progression' (c). Primary progressive and progressive relapsing presentations are less common. on DMT right after the first episode, compared to starting treatment later on? The answer seems to be no, so there is probably no harm in waiting for the second episode. ${ }^{6}$

Once relapsing MS is established, with at least one further attack after the index event, a decision needs to be made about whether to start DMT and, if so, with which agent. In patients with aggressive relapsing disease, rapidly acquiring disability, it is a fairly straightforward decision to offer 'highly active' DMT, despite the small risks of therapy. Likewise in patients with very mild relapses, at low frequency and with good recovery, it is equally straightforward to withhold any DMT, and simply observe over time (up to $20 \%$ of patients will follow a benign course on no treatment).

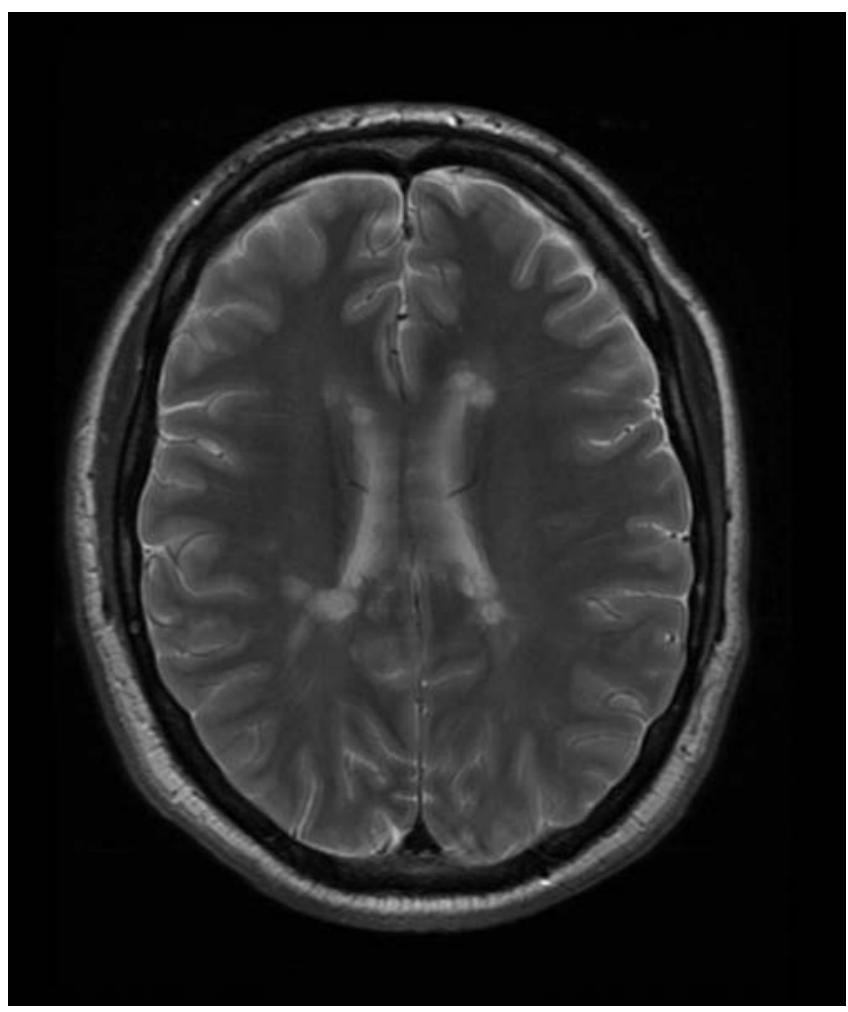

Fig 3. T2 weighted magnetic resonance imaging brain scan showing typical ovoid, periventricular multiple sclerosis lesions.

Table 1. Disease modifying treatments (DMT) in relapsing multiple sclerosis. 'Standard' DMT (shaded).

\begin{tabular}{|c|c|c|c|c|}
\hline Drug & Route & $\begin{array}{l}\text { Efficacy (relapse } \\
\text { reduction) }(\%)\end{array}$ & Toxicity & Cost pa (f) \\
\hline Interferon beta & Subcutaneous or intramuscular & 30 & $\begin{array}{l}\text { Mild; injection site reactions, deranged liver } \\
\text { function tests, depression }\end{array}$ & 7,000 \\
\hline Glatiramer acetate & Subcutaneous & 30 & Injection site reactions & 6,000 \\
\hline Mitoxantrone (unlicensed) & Monthly or quarterly infusion & 70 & $\begin{array}{l}\text { Nausea, leucopoenia, subfertility, cardiac failure, } \\
\text { leukaemia (1:300) }\end{array}$ & 1,000 \\
\hline Natalizumab & Monthly infusion & 70 & Progressive multifocal leukoencephalopathy (1:1000) & 15,000 \\
\hline Alemtuzumab (unlicensed) & Annual infusion & 90 & $\begin{array}{l}\text { Graves disease, idiopathic thrombocytopenic } \\
\text { purpura, Goodpastures syndrome, ?lymphoma }\end{array}$ & 2,000 \\
\hline
\end{tabular}


The majority of patients however, lie somewhere between these two extremes. Deciding whether to start a DMT, and if so whether 'standard' or 'highly active', depends on the treating physician's prediction of long-term prognosis. This is unfortunately difficult, with no robust markers of good or poor long-term outcome.

So what is the answer? Current thinking is for early DMT, before disability is established (and certainly before transition to a progressive course has occurred), but not too early (eg after the index event) when it is very difficult to predict how severe the long-term prognosis will be. There is probably little to lose by waiting until at least the second or third event before making a decision on starting a DMT. When that decision is made, however, increasing numbers of patients will be started on a more potent DMT from outset, or escalated to one sooner rather than later. The hope is that active suppression of early, inflammatory activity (relapses) will also delay or even prevent secondary progressive disease later on. For now though patients must be advised that there is no guarantee of this, and that more effective treatment undoubtedly comes with more risk.

\section{References}

1 Bjartmar C, Wujek JR, Trapp BD. Axonal loss in the pathology of MS: consequences for understanding the progressive phase of the disease. J Neurol Sci 2003;206:165-71.
2 Rovaris M, Confavreux C, Furlan R et al. Secondary progressive multiple sclerosis: current knowledge and future challenges. Lancet Neurol 2006;5:343-54.

3 McDonald WI, Compston A, Edan G et al. Recommended diagnostic criteria for multiple sclerosis: guidelines from the International Panel on the diagnosis of multiple sclerosis. Ann Neurol 2001;50:121-7.

4 Boggild M, Palace J, Barton P et al. Multiple sclerosis risk sharing scheme: two year results of clinical cohort study with historical comparator. BMJ 2009;339:b4677.

5 Ebers GC, Traboulsee A, Li D et al. Investigators of the 16-year longterm follow-up study. Analysis of clinical outcomes according to original treatment groups 16 years after the pivotal IFNB-1b trial. J Neurol Neurosurg Psychiatry 2010;81:907-12.

6 Kappos L, Freedman MS, Polman CH et al. BENEFIT Study Group. Long-term effect of early treatment with interferon beta-1b after a first clinical event suggestive of multiple sclerosis: 5-year active treatment extension of the phase 3 BENEFIT trial. Lancet Neurol 2009;8:987-97.

Address for correspondence: Dr M Wilson, The Walton Centre Foundation Trust, Lower Lane, Liverpool L9 7LJ.

Email: martin.wilson@thewaltoncentre.nhs.uk

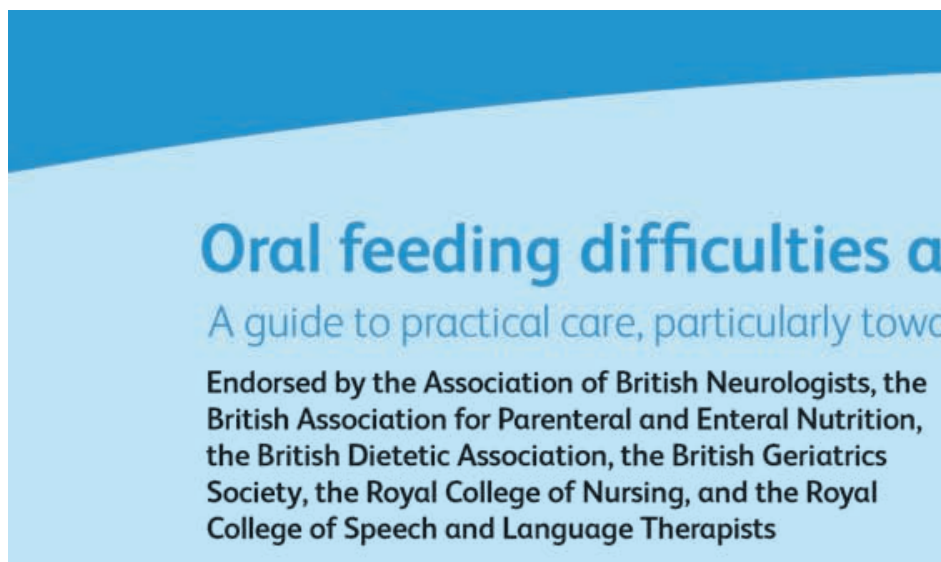

Feeding is basic to life, but it can also be an artificial medical procedure in the power of health professionals. Sometimes it causes dilemmas and strong differences of opinion between patients, relatives and professionals. This report addresses these fundamental issues.

It acknowledges the confusion and uncertainty that sometimes surround decision making and practice, including the difficulties of carrying out some of the technical interventions involved.

\section{Working party report}

The report provides evidence-based guidance on the mechanisms and techniques of oral and artificial nutrition in health and disease. It sets out the ethical and legal concerns that provide the framework for decision making. Case studies then illustrate dilemmas and solutions, for example on deciding whether to withhold or provide artificial nutrition.

This report is essential reading for all those involved in caring for people who have nutritional and oral feeding difficulties, including gastroenterologists, ward nurses, geriatricians, dietitians, speech therapists, neurologists, care home and community nurses, as well as carers, families and the patients themselves.

Published: January 2010 ISBN: 9781860163715

Price: $£ \mathbf{2 0 . 0 0}$ UK, $£ 22.00$ overseas (inc post and packing) Q.7. of Physicians}

